\title{
Endometrial lymphoid tissue: an immunohistological study
}

\author{
HUGH MORRIS, ${ }^{*}$ JOHN EDWARDS,$\dagger$ ANDREW TILTMAN, ${ }^{*}$ MALCOLM EMMS $*$
}

From the Departments of ${ }^{*}$ Pathology and $\dagger$ Obstetrics and Gynaecology, University of Cape Town Medical School and Groote Schuur Hospital, Cape Town, South Africa

SUMMARY Lymphoid tissue of the endometrium was analysed by histological, immunohistological, and electron microscopical methods in 10 healthy uteri. A panel of monoclonal antibodies recognising macrophages (OKMI), HLA-DR antigen, B lymphocytes, T lymphocytes and their subsets, and dendritic reticulum cells was used in a two stage indirect immunoperoxidase labelling technique. Endometrial lymphoid tissue showed a remarkably consistent pattern of labelling in all cases. Lymphoid tissue was present in three sites: namely, (i) intraepithelial lymphocytes (predominantly $\mathrm{T}$ lymphocytes with occasional macrophages) associated with periglandular and sub-epithelial HLA-DR+, OKMI+ macrophages; (ii) interstitial lymphocytes and macrophages; (iii) lymphoid aggregates in the stratum basalis. These were composed mainly of $\mathrm{T}$ lymphocytes with a few B lymphocytes. Dendritic reticulum cells were found in those occasional lymphoid aggregates in which germinal centres were present.

These features suggest that endometrial lymphoid tissue has many of the hallmarks of mucosal associated lymphoid tissue as found elsewhere in the body-for example, the bronchus and intestine. Endometrial lymphoid tissue appears to be unique, however, in that most of the stratum functionalis in which it is situated shows cyclical shedding during the menstrual cycle.

The human endometrium is an immunologically reactive tissue which is capable of local antibody synthesis in response to specific antigen exposure. ${ }^{1}$ Immune responses of this type are normally important for maintaining the bacteriologically sterile milieu of the endometrial lumen and in particular are concerned with the recognition and eradication of ascending bacterial infections. Immune responses have also been implicated in the pathogenesis of infertility in women suffering from endometriosis ${ }^{2}$ and may be important in the process of early nidation.

Little is known of the microscopic organisation of endometrial lymphoid tissue or of the functional subtypes of cells (B lymphocytes, T lymphocytes and their subsets, and macrophages) which participate in immune responses. Nevertheless, it is well recognised that lymphoid aggregates and scattered interstitial lymphocytes are a constant feature of normal endometria. ${ }^{3}$

We have studied the histological, immunohis-

Accepted for publication 17 February 1985 tological, and electron microscopical features of endometrial lymphoid tissue in normal uteri in order to shed light on the organisation and possible function of this tissue under physiological conditions. This study has been greatly facilitated by recent developments in immunohistological labelling methods for detecting surface markers in lymphoid tissue as well as by the availability of several monoclonal antibodies. ${ }^{4}$

\section{Material and methods}

\section{MATERIAL}

Endometrial tissue was obtained from 10 patients undergoing hysterectomy for conditions confined to the cervix alone. In each case a wedge of tissue was excised from the upper uterine segment and divided for histology, immunohistology, and electron microscopy. Tissue for immunohistology was snap frozen in liquid nitrogen and stored at $-20^{\circ} \mathrm{C}$ until cryostat sections were prepared. Tissue for electron microscopy was fixed in $5 \%$ glutaraldehyde, while the remaining tissue was fixed in $10 \%$ buffered formol-saline. 
Primary monoclonal antibodies used in study

\begin{tabular}{lll}
\hline Antibody & Specificity & Source \\
\hline OKT3 & T lymphocyte common antigen & Ortho Diagnostic Systems, USA \\
OKT4 & T helper lymphocytes (some cross reactivity & Ortho Diagnostic Systems, USA \\
& with monocytes present) & 1/100 \\
OKT8 & T cytotoxic/suppressor & Ortho Diagnostic Systems, USA \\
HLA-DR & HLA-DR antigen & Dakopatts a/s, Copenhagen \\
B-cell & B lymphocyte antigen & Dakopatts a/s, Copenhagen \\
OKMI & Monocytes & Ortho Diagnostic Systems, USA \\
DRCI & Dendritic reticulum cell network in B cell & Dakopatts a/s, Copenhagen \\
& follicles & $1 / 50$ \\
\hline
\end{tabular}

In each case a detailed menstrual history was available and the age of menarche, cycle length, and duration of menstruation were within normal limits. No hormone treatment had been given to these patients. The age range was 18-49 years with a mean of 36 years.

A total of five proliferative and five secretory phase samples were studied. Each case was allocated to one of three proliferative (early, mid, or late) or three secretory (early, mid, or late) phase subgroups on the basis of standard histological criteria. ${ }^{3}$ In addition, the uteri of three infants were examined. These included a fresh stillborn infant, gestational age 39 weeks; an infant of 3 weeks, who died of diarrhoea, vomiting, and pneumonia; and a child aged 2 years, who died of pneumonia.

\section{METHODS}

\section{Histology}

Paraffin embedded sections were routinely stained with haematoxylin and eosin.

\section{Immunohistology}

Cryostat sections ( $6 \mu \mathrm{m}$ thick) were collected on to glass slides and fixed in acetone at room temperature for $10 \mathrm{~min}$.

A two stage indirect immunoperoxidase staining technique was used. The Table lists the primary monoclonal antibodies used, their sources, and the dilutions used in the primary incubation step. In each case, after incubation with the primary monoclonal antibody $(30 \mathrm{~min})$ the sections were washed in phosphate buffered saline (PBS) and incubated for 3 min with peroxidase conjugated rabbit antimouse immunoglobulin (Dakopatts a/s, Copenhagen, Denmark) to which normal human serum (diluted $1 / 20$ in PBS) had been added to a final dilution of $1 / 50$.

Development of the peroxidase reaction was performed by incubating sections with diaminobenzidine $(0.6 \mathrm{mg} / \mathrm{ml})$ and hydrogen peroxide $(0.01 \%)$ for 5-10 $\mathrm{min}$ at room temperature. The sections were then washed in tap water, counterstained with haematoxylin, and mounted for microscopical examination.

\section{Electron microscopy}

Specimens fixed in 5\% phosphate buffered glutaraldehyde were washed overnight in $0.1 \mathrm{M}$ phosphate buffer $(\mathrm{pH} 7 \cdot 2)$. Tissue was postfixed in osmium tetroxide (Palades), dehydrated in acetone, and embedded in Spurr's resin. Sections were cut on an LKB Ultratome III and stained with uranyl acetate and lead citrate. Sections were viewed in a Hitachi H-600 electron microscope. Further sections $(1 \cdot 5-$ $2.0 \mu \mathrm{m})$ were stained with basic fuchsin and methylene blue. ${ }^{5}$

\section{Results}

\section{HISTOLOGY}

Endometrial lymphoid tissue appeared to consist of lymphocytes present at three recognisable sitesnamely, intraepithelial lymphocytes, interstitial lymphocytes, and basal lymphoid aggregates. In addition, macrophages were found in all areas of the stratum functionalis and stratum basalis.

Intraepithelial lymphocytes were found in all sections and were present in both the surface epithelium and the glandular epithelium (Fig. 1a). These cells were recognised on the basis of a characteristic pericellular halo of cytoplasmic retraction and an oval or slightly convoluted nucleus with a dense nuclear chromatin pattern. Lymphocytes in the epithelium were readily distinguished from columnar lining cells, which had a more oval nucleus with a finely stippled chromatin pattern. Lymphocytes were also readily separated from pyknotic epithelial nuclei and apoptotic cells.

Interstitial lymphocytes were more difficult to discern with certainty but were best seen in plastic embedded sections. These cells were often seen loosely arranged around spiral arterioles (Fig. 1). Plastic embedded sections clearly showed the features of capillaries and arterioles in relation to these 


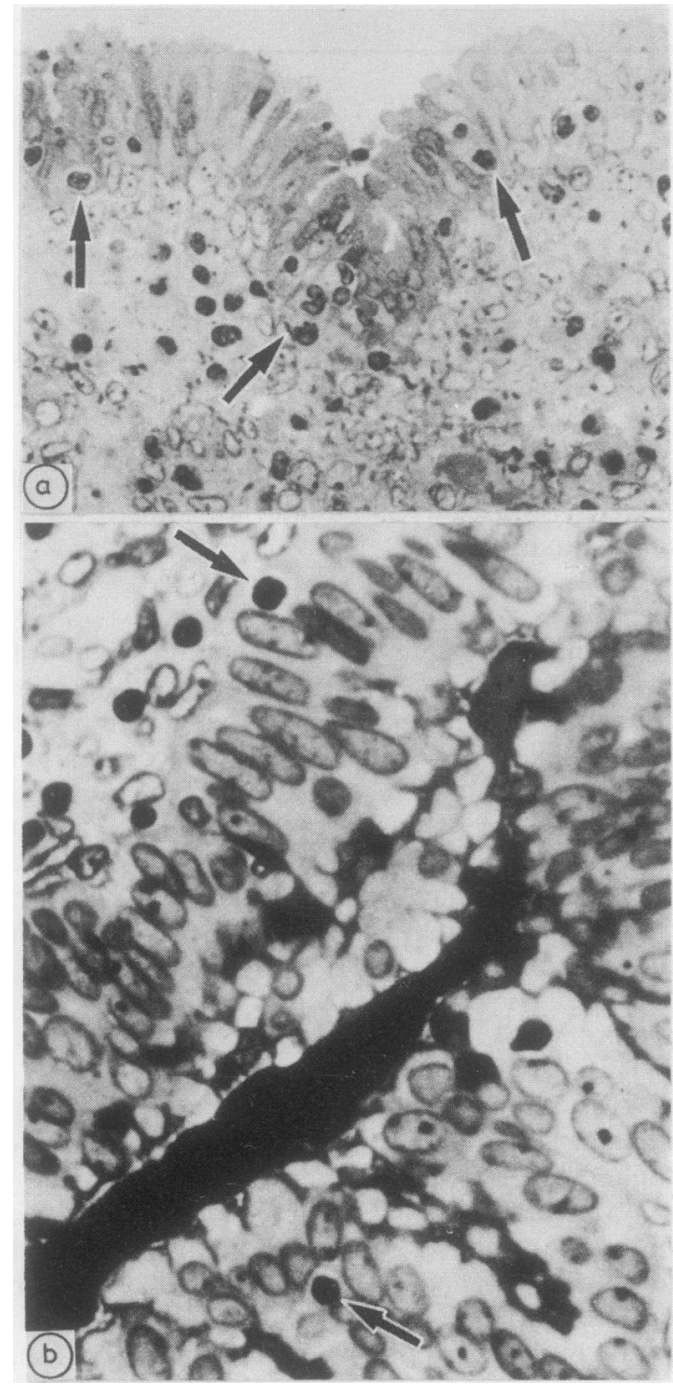

Fig. 1 (a) Endometrial surface epithelium showing intraepithelial lymphoid cells (arrows). Scattered dark cells are also present in the stroma but without immunohistological labelling it is not possible to separate lymphocytes from some stromal cells (stromal granulocytes). Plastic section $1 \mu \mathrm{m}$; dibasic stain. $\times 300$. (b) Endometrial glands in the stratum basalis showing a cluster of stromal lymphoid cells with some intraepithelial lymphocytes in the adjacent epithelium (arrows). Plastic section $1 \mu \mathrm{m}$; dibasic stain. $\times 400$.

cells. The endothelial cells had relatively abundant granular cytoplasm and in some areas migrating lymphoid cells were found apparently migrating across the capillary wall.

Lymphoid aggregates were present in all sections and were found almost exclusively in the stratum

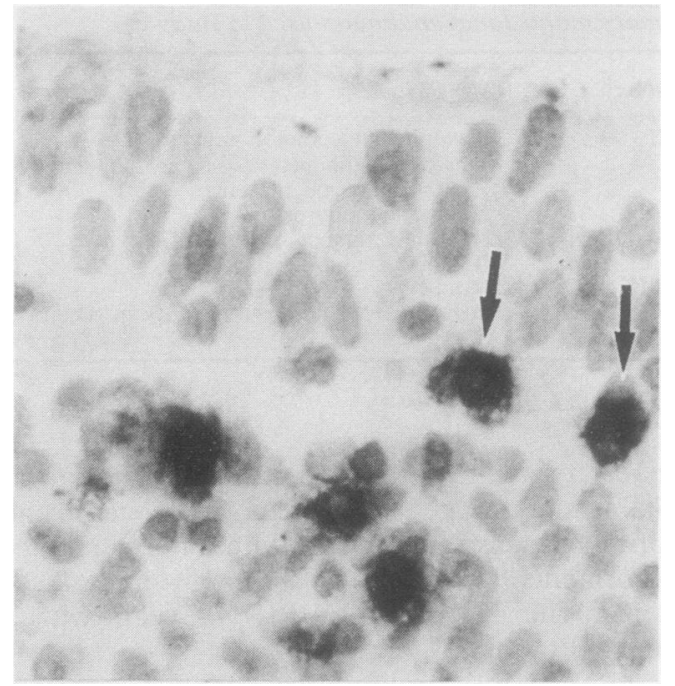

Fig. 2 Endometrial surface epithelium.

Immunoperoxidase labelling technique using monoclonal OKT8 (cytotoxic/suppressor cells) showing intraepithelial lymphocytes (arrows). Stromal lymphocytes of the same phenotype are also present. Cryostat section. $\times 800$.

basalis with only occasional aggregates in the deeper layers of the stratum functionalis (Fig. 1b). Basal lymphoid aggregates also tended to be clustered adjacent to the basal region of endometrial glands and in some cases resulted in distortion of the gland margin. In other areas lymphocytes were present in increased numbers within the glandular epithelium itself (epitheliotrophic spill over). Macrophages were often difficult to distinguish from stromal cells in haematoxylin and eosin stained sections but were more easily recognised in plastic embedded sections.

The columnar epithelial cells adjacent to intraepithelial lymphocytes were carefully scrutinised in all sections in order to search for epithelial cells which showed similarity to the specialised microvillous cells (or M cells) of the intestine. ${ }^{6}$ No convincing distinguishing features were seen on light microscopical examination of paraffin or cryostat sections, but plastic embedded sections did show some epithelial cells distorted around intraepithelial lymphocytes (see below, under electron microscopy).

\section{IMMUNOHISTOLOGY}

The above histological features were more clearly delineated in sections stained by immunohistological methods.

Intraepithelial lymphocytes were present in both surface and glandular epithelium and consisted 


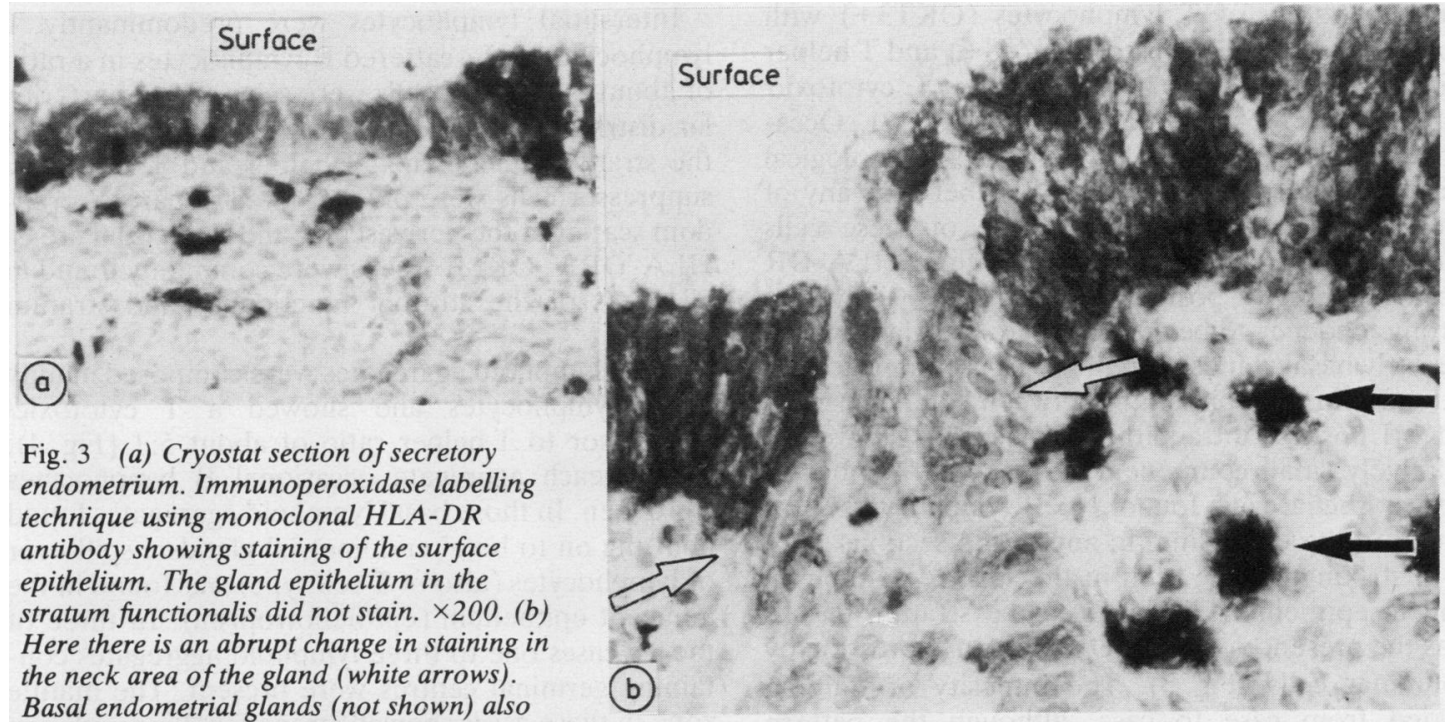

show variable degrees of $H L A-D R$

expression. HLA-DR positive stromal

lymphoid cells are also shown (black arrows). $\times 500$.

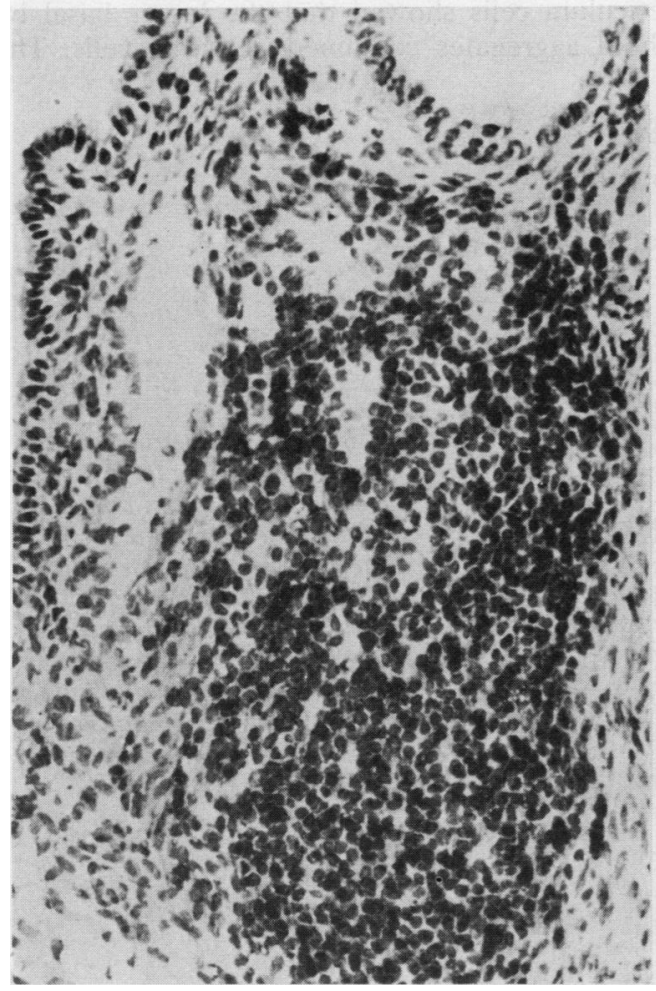

Fig. 4 Cryostat section of basal lymphoid aggregate. Immunoperoxidase labelling technique using OKT8 (T cytotoxic/suppressor lymphocytes) showing strong staining of lymphoid cells. $\times 450$.

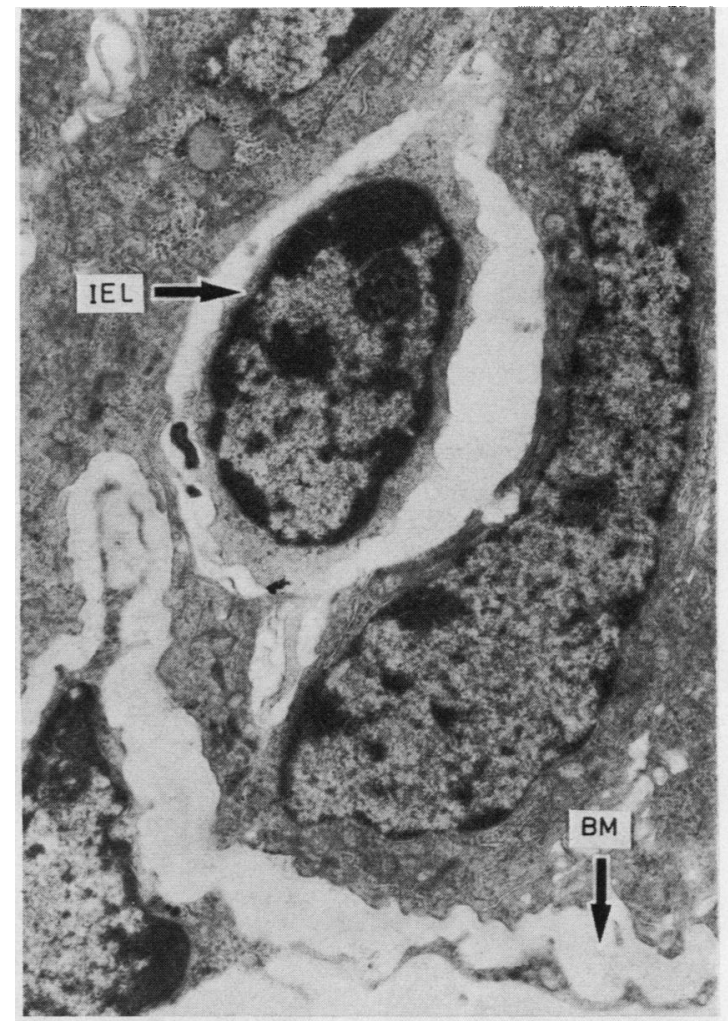

Fig. 5 Electron micrograph of endometrial gland showing single intraepithelial lymphocyte (IEL) surrounded by a retraction halo. $\times 18000$. 
almost entirely of $T$ lymphocytes (OKT3+) with both $\mathrm{T}$ cytotoxic/suppressor (OKT8+) and T helper lymphocytes (OKT4) present (ratio $\mathrm{T}$ cytotoxic/ suppressor to $T$ helper about 4:1) (Fig. 2). Occasional intraepithelial cells with typical histological features of lymphocytes failed to label with any of the $T$ cell antibodies. The nature of these cells remains to be determined. In addition, HLA-DR positive, OKMI positive macrophages showed a strong tendency to be dispersed around glands (particularly basal glands) and in some cases appeared to extend into the epithelium between columnar cells. OKMI positive intraepithelial macrophages were a relatively small component of the total number of intraepithelial cells found. No B lymphocytes were found in the epithelium in any of the sections.

A striking change seen in the surface epithelium and in epithelium of glands in the stratum basalis was the presence of HLA-DR antigen expression by columnar cells (Fig. 3). The intensity of staining varied from case to case, although the pattern remained consistent. At the necks of the glands the epithelial cells showed a distinct change in the intensity of labelling with a complete absence of staining in the main segments of the glands (Fig. 3). In the basal region of each gland variable expression of the antigen was again found.
Interstitial lymphocytes were predominantly $\mathrm{T}$ lymphocytes with scattered B lymphocytes in a ratio of about $6: 1$. The $T$ cells were seen in a periarteriolar distribution as well as diffusely in the stroma of the stratum functionalis. $T$ helper and $T$ cytotoxic/ suppressor cells were present in an apparently random scatter in the perivascular and interstitial areas. HLA-DR +, OKMI+ cells were commonly found in all areas of the stratum functionalis and stratum basalis.

Basal lymphoid aggregates were composed mainly of $\mathbf{T}$ lymphocytes and showed a $\mathrm{T}$ cytotoxic/ suppressor to $\mathrm{T}$ helper ratio of about 5:1 (Fig. 4). Within each aggregate occasional B lymphocytes were seen. In those basal lymphoid aggregates found abutting on to basal endometrial glands a spill over of lymphocytes (also of T cell type) was found in the adjacent epithelium (epitheliotropism). In three of the 10 cases one to three lymphoid aggregates containing germinal centres were present. The mantle zone in these cases showed strong labelling with pan B cell antibody, with a lesser degree of staining of the germinal centre itself. This pattern is typically seen in reactive lymphoid aggregates in lymph nodes and tonsil. ${ }^{7}$ Immunolocalisation of dendritic reticulum cells showed that the larger basal lymphoid aggregates contained dendritic cells. These

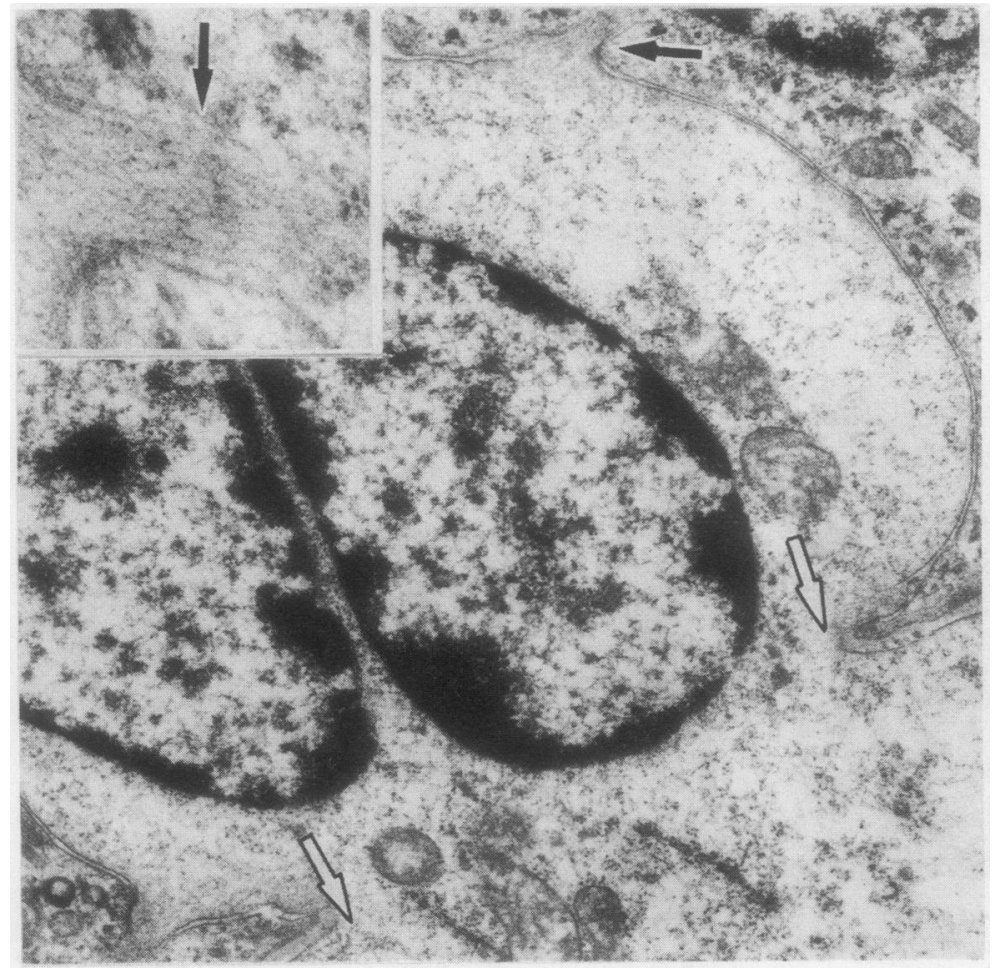

Fig. 6 (a) Endometrial gland showing a migrating large lymphoid cell with cytoplasm on both sides of the basement membrane (between white arrows). The presence of phagocytic material and a bilobed nucleus suggests that this cell may be a macrophage. The black arrow shows the presence of a cell junction $\times 15$ 000. Inset: High power view of the cell junction (black arrow). $\times 60000$. 


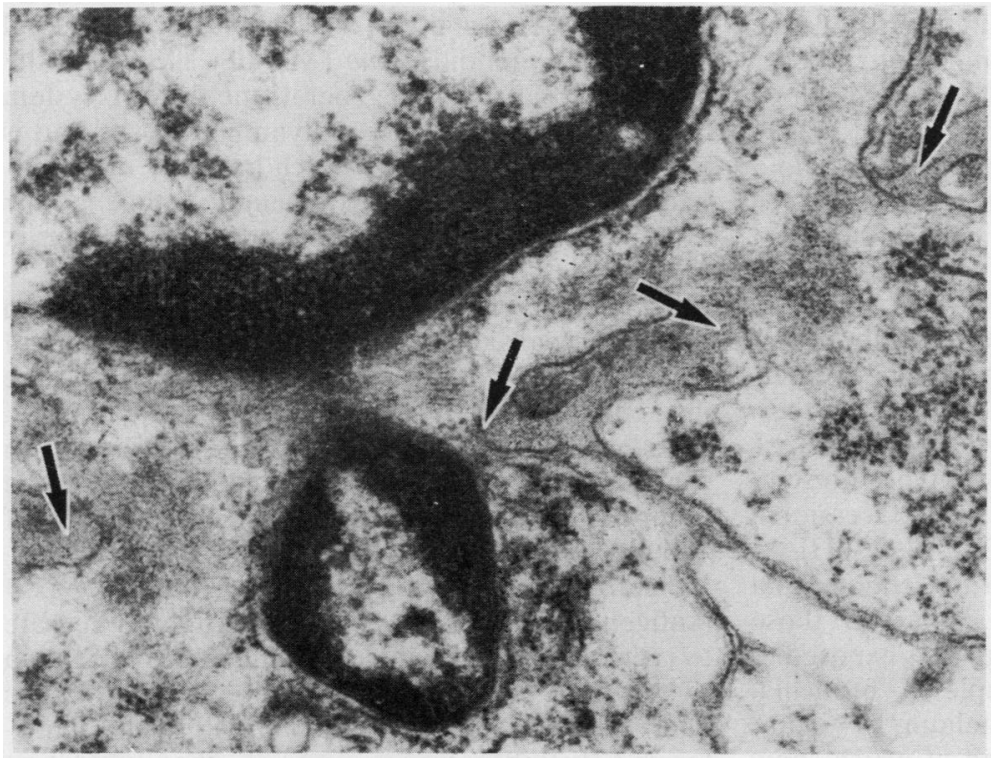

Fig. 7 Endometrial gland showing an intraepithelial lymphoid cell with evidence of migration across the basement membrane between the areas marked with dark arrows. $\times 4500$.

were present in the form of a prominent network situated within and between the lymphocytes of each aggregate.

\section{ELECTRON MICROSCOPY}

Intraepithelial lymphocytes were delineated in the surface and gland epithelium in all cases examined. The nuclear chromatin pattern and the size of the nucleus as well as the presence of a retraction halo surrounding the cell readily separated intraepithelial lymphocytes from adjacent columnar cells (Fig. 5). A striking feature of intraepithelial lymphocytes and macrophages was the presence of irregular cytoplasmic processes which extended between columnar cells and in many cases indented adjacent columnar cells. In addition, at these sites of invagination the plasma membrane of intraepithelial lymphocytes and columnar cells were directly opposed. These contacts took the form of slender cytoplasmic processes, which in some areas showed blurring of membranes at the point of apposition (Fig. 6). Intraepithelial macrophages showed more abundant cytoplasm, secondary phagolysomes, mitochondria, and rough endoplasmic reticulum. These cells were morphologically suggestive of macrophages and presumably correspond to the light microscopical appearance of OKMI positive cells in the epithelium. In the late secretory phase some macrophages appeared to contain apoptotic debris in phagosomes. These cells were also found in the adjacent stroma.

Interstitial lymphocytes were difficult to delineate without the assistance of immunological markers.
Occasional cells, however, with the dense nuclear chromatin pattern characteristic of lymphocytes, were seen in the interstitium and surrounding small blood vessels. Interstitial lymphocytes apparently in the process of migration across the basement membrane into the surface or gland epithelium were seen (morphological evidence of migration was based on the direction of basement membrane displacement, the amount of cytoplasm at the advancing front of the cell compared with the trailing end, and the position of nuclear pinching at the point of migration across the basement membrane) (Fig. 7).

\section{ENDOMETRIAL LYMPHOID TISSUE IN}

PROLIFERATIVE AND SECRETORY PHASES

All sections were categorised into groups for comparison-that is, proliferative $v$ secretory phase. In the early proliferative (early postmenstrual) phase basal aggregates as well as intraepithelial lymphocytes were present in all cases. In the remainder of the proliferative and early and midsecretory phases no substantial alteration in the number of lymphoid cells was found. The late secretory phase, however, was characterised by increased numbers of leucocytes in the stroma (recognised on the basis of increased amounts of endogenous peroxidase) and, although we have not quantitated the total number of lymphocytes in our material, there appeared to be increased numbers of stromal lymphocytes in the late secretory phase.

INFANT UTERI

Histologically, all three cases showed inactive 
endometria with occasional halo cells in the epithelium of the surface epithelium and, to a lesser extent, the gland epithelium. Immunohistology showed the presence of occasional $T$ lymphocytes in the epithelium and the stroma, but no lymphoid aggregates were found in the basal regions.

\section{Discussion}

In this study we have documented the distribution of $T$ and $B$ lymphocytes and macrophages in endometrial lymphoid tissue. The predominant intraepithelial lymphocytes are $T$ lymphocytes with both $T$ cytotoxic/suppressor and $\mathrm{T}$ helper subtypes present. No intraepithelial B cells were seen. Occasional intraepithelial macrophages (OKTM + , HLA$\mathrm{DR}+$ ) were also a feature of normal endometrial epithelium. These findings are similar to those obtained in studies of other mucosal sites exposed to the environment-for example, bronchial epithelium, ${ }^{8}$ intestinal epithelium, ${ }^{6}$ skin, ${ }^{9}$ oesophagus, ${ }^{10}$ and cervix uteri, ${ }^{11}$ all of which show intraepithelial lymphocytes of mainly $\mathrm{T}$ cell type.

A striking feature in all cases was the presence of lymphoid aggregates in the stratum basalis, which consists predominantly of $T$ lymphocytes with $T$ cytotoxic/suppressor cells exceeding $T$ helper cells. This was an unexpected finding as we anticipated that lymphocyte aggregates which were devoid of germinal centres would consist largely of B lymphocytes (as is usually seen in unstimulated primary follicles in lymph nodes). This difference may be explained on the basis of the limited antigenic stimulation which appears to exist in the endometrial cavity since this area is normally a bacteriologically sterile environment. It is well recognised, however, that it is possible for particulate matter to be transported from the vaginal and the endocervical lumina to as high as the fallopian tube lumen. ${ }^{12}$ Hence there is a potential channel for bacterial antigens to gain access to both the endometrial cavity and the tubal lumen. In those lymphoid aggregates which showed germinal centres the mantle zone showed characteristic B lymphocyte staining, suggesting that unstimulated lymphoid aggregates may transform to secondary follicles with germinal centres on exposure to appropriate antigens. An analogous situation is found in the ontogeny of Peyer's patches in animals. In the rat, neonatal Peyer's patches consist mainly of $T$ lymphocytes but on exposure to feeds, and therefore various antigens, B lymphocyte proliferation occurs and germinal centres are formed.

The above findings (intraepithelial lymphocyte of a particular lymphocyte type, stromal macrophages and lymphocytes in the interstitial areas, and basal lymphoid aggregates) suggest that endometrial lym- phoid tissue has many of the hallmarks of mucosal associated lymphoid tissue (MALT) as seen at the other mucosal sites. In general, the MALT system forms part of the peripheral lymphoid tissue and is present at epithelial sites which are fully or potentially exposed to environmental antigens-for example, bronchus, intestine, salivary ducts, cervix uteri.

The characteristic features of the MALT system have been partially elucidated and include many of those presented in this report. It is thought that the intraepithelial lymphocytes are responsible for antigen detection in columnar epithelia (facilitated in some areas by specialised columnar $\mathrm{M}$ cells), ${ }^{6}$ while other accessory cells are important in the uptake of antigen in squamous epithelia-for example, Langerhans' cells in the oesophagus ${ }^{10}$ and cervix." Antigen primed lymphocytes are thought to migrate to regional lymphoid tissue (lymph nodes) and then to enter the systemic circulation (via the thoracic duct) en route to the central lymphoid tissue. Transformed lymphocytes re-entering the circulation from the central lymphoid tissue show a strong tendency to home back to the area of original antigen encounter. ${ }^{4}$

In the endometrium an immune response of this type is also consistent with the data on local immunoglobulin production by endometrium.' The mechanisms and possible routes of lymphocyte traffic from the areas of antigen encounter-for example, intraepithelial lymphocytes or macrophages in surface or gland columnar epitheliumto regional lymphoid tissue (regional pelvic lymph nodes) are not, however, apparent from our study. Nevertheless, two possible mechanisms deserve further consideration. Firstly, it is possible that some columnar cells act as accessory cells and enhance antigen uptake by intraepithelial lymphocytes and macrophages. This suggestion is based on our finding on electron microscopy of close apposition and, in some cases, blurring of the plasma membrane between intraepithelial lymphocytes and columnar cells. Secondly, it is possible that these antigen primed lymphocytes and macrophages migrate to the lymphoid aggregates in the stratum basalis. It is well recognised that dendritic reticulum cells are antigen presenting cells and are important in the process of antigen specific B lymphocyte transformation. ${ }^{7}$ In this way amplification of the immune response could occur within the basal lymphoid aggregates. This would in turn be followed by the migration of lymphocytes to regional and then central lymphoid tissue.

Since the stratum basalis remains after shedding of the stratum functionalis during menstruation it is likely that the basal lymphoid aggregates are a rela- 


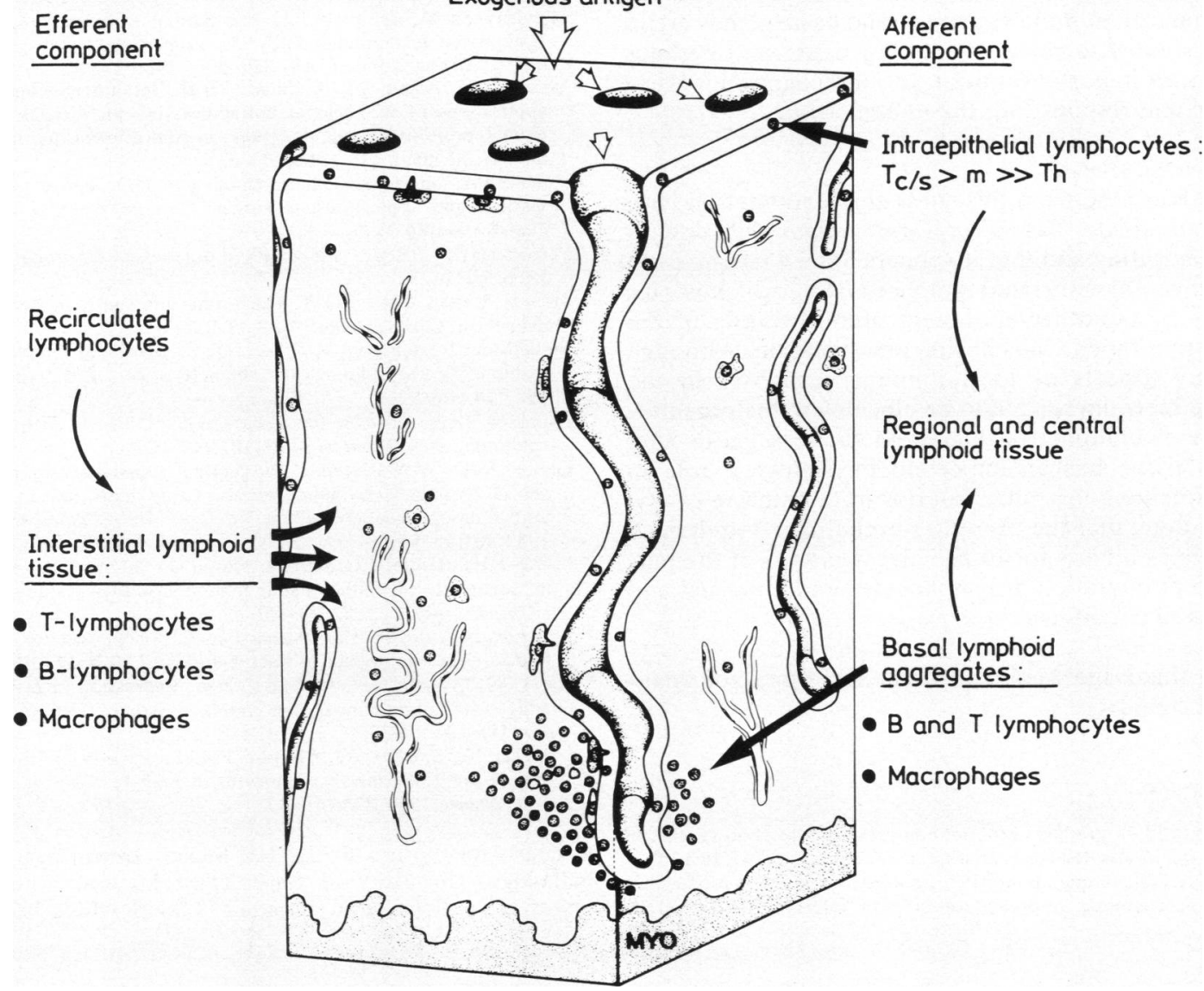

Fig. 8 Diagram summarising the organisation of endometrial lymphoid tissue. Lymphoid cells are found at three main sites-namely, (i) intraepithelial lymphocytes, in which T cytotoxic/suppressor cells exceed Thelper cells and macrophages; (ii) interstitial lymphoid tissue; (iii) basal lymphoid aggregates, in which most cells are of T cytotoxic/suppressor phenotype apart from those aggregates which show germinal centre formation (in which $B$ cells predominate and dendritic reticulum cells are found). Lymphocyte traffic remains speculative but is likely to entail the migration of intraepithelial lymphocytes and basal lymphocytes to regional lymph nodes (afferent component). Amplification of the immune response may occur in the central lymphoid tissue and recirculated lymphocytes probably home back to the area of the endometrium in which antigen was originally encountered (efferent component). $T_{c / s}=T$ cytotoxic/suppressor lymphocyte phenotype. M $=$ macrophages. $T_{h}=T$ helper lymphocyte phenotype. $M Y O=$ myometrium.

tively constant component of endometrial lymphoid tissue and indeed may act as replenishment centres during the early proliferative (early postmenstrual) phase. These speculative views are summarised in Fig. 8.

It is also of interest to compare the pattern of HLA-DR staining by columnar epithelial cells with the distribution of lymphoid tissue. HLA-DR staining was present (albeit of variable intensity) in the surface epithelial cells and in the epithelium of the glands situated in the stratum basalis. Although orig- inally thought to be confined to lymphoid cells (macrophages, B lymphocytes, and activated $T$ cells), expression of HLA-DR antigen by normal epithelial cells is now documented at several sites including ductal epithelium of the breast ${ }^{13}$ and gland epithelium of the endometrium (Natali et $a^{13}$ and present observations). HLA-DR antigen is also expressed in epithelia in which there is an active local immune response (such as graft versus host disease in the epidermis ${ }^{13}$ ). The findings in our study therefore suggest that areas of increased HLA-DR 
expression in epithelial cells are also areas of enhanced immune response. The basal glands are in an area of increased lymphoid tissue and perhaps play an important, but as yet unrecognised, part in immune responses in the endometrium.

\section{CONCLUSION}

We conclude from this study that endometrial lymphoid tissue has a uniform immunohistological organisation and that it appears to be a unique form of mucosal associated lymphoid tissue which is capable of rapid replenishment after shedding of the stratum functionalis during menstruation. Although many aspects of local immune responses in the endometrium remain to be elucidated, the organisation of endometrial lymphoid tissue suggests that this tissue has an important physiological role in maintaining the mileu interior of the uterine cavity. We hope that the present morphological study may serve as a basis for future investigations of the pattern of migration of lymphocytes under normal and disease conditions.

We thank the Medical Research Council for financial support.

\section{References}

' Ogra PL, Ogra SS. Local antibody response to polio vaccine in the human female genital tract. J Immunol 1973;110:1307.

${ }^{2}$ Weed JC, Arquembourg PC. Endometriosis: can it produce an autoimmune response resulting in infertility? Clin Obstet
Gynecol 1980;23:885-93.

${ }^{3}$ Hendricksen M, Kempson RL. The normal endometrium. In: Bennington JL, Saunders WB, eds. Surgical pathology of the uterine corpus. London: WB Saunders, 1980:36-98.

4 Mason DY, Naiem M, Abdulaziz, et al. Immunohistological applications of monoclonal antibodies. In: McMichael AJ, Fabe J, eds. Monoclonal antibodies in clinical medicine. London: Academic Press, 1983:585-635.

${ }^{5}$ Agnese PA, Jensen K. Dibasic staining of large epoxy tissue sections and applications to surgical pathology. Am J Clin Pathol 1984;80:25-9.

- Parrott DMV. The gut as a lymphoid organ. Clin Gastroenterol 1976;5:211-28.

' Stein H, Gerdes J, Mason DY. The normal and malignant germinal centre. Clin Haematol 1982;11:531-59.

${ }^{8}$ Bienenstock J, Johnson M, Perey DYE. Bronchial lymphoid tissue. (I) Morphological characteristics. Lab Invest 1973;28:686-92.

'Patterson JAK, Edelson RL. Interaction of T-cells with the epidermis. Br J Dermatol 1982; 107:107-22.

${ }^{10}$ Geboes K, De Wolf-Peeters C, Rutgeerts P, Janssen J, van Trappen G, Desmet V. Lymphocytes and Langerhans' cells in the human oesophageal epithelium. Virchows Arch (Pathol Anat) 1983;401:45-55.

" Morris HHB, Gatter KC, Stein H, Mason DY. Langerhans' cells in human cervical epithelium: an immunohistological study. $\mathrm{Br}$ J Obstet Gynaecol 1983;90:400-11.

12 Egli GE, Newton M. The transport of carbon particles in the human female reproductive tract. Fertil Steril 1961;12:151-5.

${ }^{13}$ Natal PG, Martino C, Quaranta V, et al. Expression of Ia-like antigens in normal human non-lymphoid tissues. Transplantation 1981;31:75-78.

${ }^{14}$ Lampert IA, Suitters AJ, Chisolm PM. Expression of Ia-like antigen on epidermal keratinocytes in graft-versus-host disease. Nature 1981;293:149-50.

Requests for reprints to: Dr HB Morris, Department of Pathology, University of Cape Town Medical School, Observatory, Cape Town, Republic of South Africa 7925. 